Publ. Mat. (2014), 37-48

Proceedings of New Trends in Dynamical Systems. Salou, 2012.

DOI: 10.5565/PUBLMAT_Extra14_02

\title{
ON THE INTEGRABILITY OF SOME THREE-DIMENSIONAL LOTKA-VOLTERRA EQUATIONS WITH RANK-1 RESONANCES
}

\author{
Waleed Aziz And Colin Christopher
}

To Jaume, in much appreciation of his support and kindness over the years

Abstract: We investigate the local integrability in $\mathbb{C}^{3}$ of some three-dimensional Lotka-Volterra equations at the origin with $(p: q: r)$-resonance,

$$
\begin{aligned}
& \dot{x}=P=x(p+a x+b y+c z), \\
& \dot{y}=Q=y(q+d x+e y+f z), \\
& \dot{z}=R=z(r+g x+h y+k z) .
\end{aligned}
$$

Recent work on this problem has centered on the case where the resonance is of "rank-2". That is, there are two independent linear dependencies of $p, q$ and $r$ over $\mathbb{Q}$. Here, we consider some situations where there is only one such dependency. In particular, we give necessary and sufficient conditions for integrability for the case of $(i,-i, \lambda)$-resonance with $\lambda \notin i \mathbb{R}$ (after a scaling, this is just the case $p+q=0$ with $q / r \notin \mathbb{R})$, and also the case of $(i-1,-i-1,2)$-resonance (a subcase of $p+q+r=0$ ) under the additional assumption that $a=k=0$.

Our necessary and sufficient conditions for integrability are given via the search for two independent first integrals of the form $x^{\alpha} y^{\beta} z^{\gamma}(1+O(x, y, z))$. However, a new feature in the case of rank- 1 resonance is that there is a distinguished choice of analytic first integral, and hence it makes sense to seek conditions for just one (analytic) first integral to exist. We give necessary and sufficient conditions for just one first integral for the two families of systems mentioned above, except that for the second family some of the cases of sufficiency have been left as conjectural.

2010 Mathematics Subject Classification: 34C20.

Key words: Integrability, Lotka-Volterra, Darboux, Jacobi multiplier.

\section{Introduction}

The purpose of this paper is to extend some recent work $[\mathbf{2}, \mathbf{3}]$ on the local integrability in $\mathbb{C}^{3}$ at the origin of the three-dimensional LotkaVolterra equations

$$
\begin{aligned}
& \dot{x}=P=x(p+a x+b y+c z), \\
& \dot{y}=Q=y(q+d x+e y+f z), \\
& \dot{z}=R=z(r+g x+h y+k z),
\end{aligned}
$$


to more general resonances. Related work on the integrability of LotkaVolterra and other three dimensional systems can be found in $[\mathbf{1}, \mathbf{4}, \mathbf{6}$, $7,8,9,10,11,12,13,14]$.

Recall that system (1) is integrable at the origin if there exists a change of coordinates

$$
X=x(1+O(x, y, z)), \quad Y=y(1+O(x, y, z)), \quad Z=z(1+O(x, y, z)),
$$

bringing (1) to a system orbitally equivalent to the linear system:

$$
\dot{X}=p X m, \quad \dot{Y}=q Y m, \quad \dot{Z}=r Z m,
$$

where $m=m(X, Y, Z)=1+O(X, Y, Z)$. This is equivalent to the existence of two first integrals of the form

$$
\phi_{1}=x^{r_{1}} y^{s_{1}} z^{t_{1}}(1+O(x, y, z)), \quad \phi_{2}=x^{r_{2}} y^{s_{2}} z^{t_{2}}(1+O(x, y, z)),
$$

where $\left(r_{1}, s_{1}, t_{1}\right) \times\left(r_{2}, s_{2}, t_{2}\right) \neq 0$. If the change of coordinates can be chosen so that $m \equiv 1$, then we say the system is linearizable.

In the works cited above, the resonances at the origin have all been of "rank-2". That is, there are two independent linear dependencies of $p, q$ and $r$ over $\mathbb{Q}$. This condition is satisfied if and only if we can rescale the system so that $p, q$ and $r$ are in $\mathbb{Z}$. Necessary and sufficient conditions for integrability can therefore be obtained via the search for two independent first integrals of the form $x^{\alpha} y^{\beta} z^{\gamma}(1+O(x, y, z))$, with $\alpha, \beta$ and $\gamma$ in $\mathbb{Z}$. In the case where $p, q$ and $r$ do not all share the same sign we can reduce our considerations to the search for two analytic first integrals.

Our aim here is to consider the integrability of the origin of (1) in the case where there resonance is of rank-1. That is, there is only one linear dependency of $p, q$ and $r$ over $\mathbb{Q}$. As above, to prove integrability, we still seek two first integrals of the form $x^{\alpha} y^{\beta} z^{\gamma}(1+O(x, y, z))$, but at most one of these can be analytic in this case.

In particular, we give necessary and sufficient conditions for the origin of system (1) to be integrable in the case of $(i:-i: \lambda)$-resonance for $\lambda \notin i \mathbb{R}$, and also for the case of $(i-1:-i-1: 2)$-resonance under the additional assumption that $a=k=0$. The first case corresponds, after a scaling, to the condition $p+q=0$ with $q / r \notin \mathbb{R}$. The latter case is a subset of those systems with $p+q+r=0$. Unfortunately, the calculations for more general systems with $p+q+r=0$ were beyond our computational resources, and even in this subcase, the assumption of $a=k=0$ was necessary to make the computations tractable. We 
do not envisage that the more general cases will bring forth any essentially different phenomena to the ones found here, although it would be interesting to see if these computations could be pursued further.

A new feature in the case of rank-1 resonances in the Siegel domain is that there is a distinguished choice of analytic first integral of the form $x^{\alpha} y^{\beta} z^{\gamma}(1+O(x, y, z))$, and hence it makes sense to seek conditions for just one (analytic) first integral to exist. We give necessary and sufficient conditions for just one first integral in the cases mentioned above, except that for $(i-1,-i-1,2)$-resonance, a few cases of sufficiency have been left as conjectural.

\section{Definitions}

Let

$$
X=P \frac{\partial}{\partial x}+Q \frac{\partial}{\partial y}+R \frac{\partial}{\partial z}
$$

be the associated vector field to system (1). Given a polynomial $F \in$ $\mathbb{C}[x, y, z]$, a surface $F=0$ is called an invariant algebraic surface of system (1), if the polynomial $F$ satisfies the equation

$$
\dot{F}=X F=P \frac{\partial F}{\partial x}+Q \frac{\partial F}{\partial y}+R \frac{\partial F}{\partial z}=C_{F} F
$$

for some polynomial $C_{F} \in \mathbb{C}[x, y, z]$. Such a polynomial is called the cofactor of the invariant algebraic curve $F=0$. One can note that from equation (3) that any cofactor has at most degree one since the polynomial vector field has degree two.

To complete the study of integrals of parametric families, we will also need the notion of exponential factor which plays the same role as an invariant algebraic surface in the case when two such surfaces coalesce. Let $E(x, y, z)=\exp (f(x, y, z) / g(x, y, z))$ where $f, g \in \mathbb{C}[x, y, z]$, then $E$ is an exponential factor if

$$
X E=C_{E} E,
$$

for some polynomial $C_{E}$ of degree at most one. The polynomial $C_{E}$ is called the cofactor of $E$.

A Darboux function is a function of the form

$$
D=\prod F_{i}^{\lambda_{i}} E^{\lambda_{0}}
$$

where the $F_{i}$ are invariant algebraic surfaces of the system, and $E=$ $\exp (f / g)$ is an exponential factor. Given a Darboux function, $D$, we can compute

$$
X(D)=D\left(\sum \lambda_{i} C_{F_{i}}+\lambda_{0} C_{E}\right)
$$


Clearly, the function $D$ is a non-trivial first integral of the system if and only if the cofactors $C_{F_{i}}$ and $C_{E}$ are linearly dependent.

For Darboux integrability in two dimensions, we seek a Darboux function which is either a first integral or integrating factor for the system. From the latter, it is possible to find a first integral by quadratures.

In higher dimensions, the role of the integrating factor is taken by the Jacobi multiplier. In the context of Darboux integrability, we usually consider the corresponding reciprocals: inverse integrating factors, and inverse Jacobi multipliers [5]. A function $M$ is an inverse Jacobi multiplier for the vector field $X$ if it satisfies the equation

$$
X(M)=M \operatorname{div}(X) \quad \Longleftrightarrow \operatorname{div}(X / M)=0 .
$$

A Darboux inverse Jacobi multiplier, $D$, must satisfy $\lambda_{i} C_{F_{i}}+\lambda_{0} C_{E}=$ $\operatorname{div}(X)$.

In three dimensions, the existence of two independent first integrals implies the existence of an inverse Jacobi multiplier. Conversely, given just one first integral, $\phi$, and an inverse Jacobi multiplier, $M$, one can construct another first integral by integrating along the level surfaces of the first integral, noting that $M$ gives rise to an integrating factor on each level surface.

Unfortunately, this prescription breaks down near a critical point, where the leaves become singular. However, the following theorem allows us to construct a second first integral at a critical point in many cases. We use the usual multi-index notation $X^{I}=x^{i} y^{j} z^{k}$ to simplify the notation.

Theorem 1. Suppose the analytic vector field

(5) $x\left(\lambda+\sum_{|I|>0} A_{x I} X^{I}\right) \frac{\partial}{\partial x}+y\left(\mu+\sum_{|I|>0} A_{y I} X^{I}\right) \frac{\partial}{\partial y}+z\left(\nu+\sum_{|I|>0} A_{z I} X^{I}\right) \frac{\partial}{\partial z}$

has a first integral $\phi=x^{\alpha} y^{\beta} z^{\gamma}(1+O(x, y, z))$ with at least one of $\alpha, \beta, \gamma \neq 0$ and a Jacobi multiplier $M=x^{r} y^{s} z^{t}(1+O(x, y, z))$ and suppose that the cross product of $(r-i-1, s-j-1, t-k-1)$ and $(\alpha, \beta, \gamma)$ is bounded away from zero for any integers $i, j, k \geq 0$. Then the system has a second analytic first integral of the form $\psi=x^{1-r} y^{1-s} z^{1-t}(1+$ $O(x, y, z))$, and hence system (5) is integrable.

Proof: See [2]. 
We note finally that it is sometimes possible to reduce the critical point to one in the Poincaré domain. That is, the origin does not lie in the convex hull of the eigenvalues of the critical point. In this case, it is only necessary to check that a finite number of resonant terms vanish in the normal form to conclude that the system is linearizable.

We make use of this fact in two ways: either by decoupling two of the three equations of (1) to get one in the Poincaré domain; or performing a blow-down to a critical point in the Poincaré domain. In the former case, ad-hoc arguments are used to show that the third equations can also be linearized. In the latter, we can pull back the two first integrals of the linear system to first integrals of the original system.

\section{Systems with $(i:-i: \lambda)$-resonance}

In this section we study the local integrability of the origin for the three dimensional Lotka-Volterra equations,

$$
\begin{aligned}
& \dot{x}=P=x(i+a x+b y+c z), \\
& \dot{y}=Q=y(-i+d x+e y+f z), \\
& \dot{z}=R=z(\lambda+g x+h y+k z),
\end{aligned}
$$

with $\lambda \notin i \mathbb{R}$.

Necessary conditions for the existence of one first integral were found by searching for a first integral of the form $x y(1+O(x, y, z))$ and, for integrability, a second first integral of the form $y^{\lambda} z^{i}(1+O(x, y, z))$. The computations were carried as far as the resonant terms in $(x y)^{2}$ to obtain the conditions below. Sufficiency of these conditions was then proved case by case. The computations were carried out in Maple.

Some care needs to be taken over the integrability conditions in this case since polynomials in $\lambda$ appear in the coefficients of the first integrals. Any value of $\lambda$ which appears as a root in these denominators will have to be checked separately. However, in the case above, these roots are purely integer multiples of $i$ and so have already been excluded.

Lemma 2. Let $X$ be the vector field associated to (6), and $X_{0}$ the restriction of $X$ onto the plane $z=0$. If $X_{0}$ is integrable (that is, it has an analytic first integral of the form $x y+\cdots)$, and $X_{0}\left(\phi_{0}(x, y)\right)=$ $f(x, y)$ for some analytic functions $\phi_{0}$ and $f$ then, for any analytic function $g(x, y, z)$, there exists an analytic function $\phi(x, y, z)=\phi_{0}(x, y)+$ $z \widetilde{\phi}(x, y, z)$ such that $X(\phi)=f(x, y)+z g(x, y, z)$.

Proof: We write

$$
X=X_{0}+z(\lambda+g x+h y) \frac{\partial}{\partial z}+z X_{1}+k z^{2} \frac{\partial}{\partial z}
$$


where $X_{1}=c x \frac{\partial}{\partial x}+f y \frac{\partial}{\partial y}$. Writing

$$
\phi(x, y, z)=\phi_{0}(x, y)+\sum_{i>0} \phi_{i}(x, y) z^{i}, \quad g=\sum_{i \geq 0} g_{i}(x, y) z^{i}
$$

then it is clear that we need to solve

$$
\left(X_{0}+(m+1)(\lambda+g x+h y)\right) \phi_{m+1}=-\left(X_{1}+k m\right) \phi_{m}+g_{m},
$$

for each $m \geq 0$. It is easy to see that there are no obstructions to obtaining a unique formal series solution for $\phi_{m}$ in this way and its convergence follows from the following argument.

Since the system $X_{0}$ is integrable, there is a change of coordinates $(x, y)=(X+o(X, Y), Y+o(X, Y))$, which brings $X_{0}$ to the form

$$
X_{0}=r(X, Y)\left(i X \frac{\partial}{\partial X}-i Y \frac{\partial}{\partial Y}\right) \text {, }
$$

for some analytic function $r(X, Y)=1+O(X, Y)$. Thus (7) is now of the form

$$
\left(i X \partial_{X}-i Y \partial_{Y}+(m+1)(\lambda+\ell)\right) \widetilde{\phi}_{m+1}=-r^{-1}\left(\widetilde{X}_{1}+k m\right) \widetilde{\phi}_{m}+\widetilde{g}_{m},
$$

where $\widetilde{\phi}_{m}(X, Y)=\phi_{m}(x, y)$ and similarly $\ell$ and $\widetilde{g}_{m}$ are just the functions $(\lambda(1-r)+g x+h y) / r$ and $g / r$ in $X$ and $Y$ coordinates (where $r$ is already defined in $X$ and $Y$ coordinates). Furthermore, $\widetilde{X}_{1}=\widetilde{P} \frac{\partial}{\partial X}+\widetilde{Q} \frac{\partial}{\partial Y}$ is just the transformation of $X_{1}$ to the coordinates $X$ and $Y$.

Let $u=X+Y+Z$ and $v=X+Y$. Choose $N$ such that $\sum \widetilde{g}_{m} z^{m}$ is majorized by $N(1-N u)^{-1}$, then $\widetilde{g}_{m}$ is majorized by $N^{m+1}(1-N v)^{-m-1}$. We write $\widetilde{\phi}_{m}$ as a sum of homogeneous terms, $\widetilde{\phi}_{m}=\sum \varphi_{m, i}$, and choose $M>N$ such that $r^{-1} \widetilde{P}, r^{-1} \widetilde{Q}, \ell$ and $r^{-1}$ are all majorized by $1 /(1-M v)$ and all the coefficients $a$ to $k$ are less than $M$ in absolute value. We also let $\kappa$ denote $|\Re(\lambda)|$, so that if $A$ majorizes $\left(i X \partial_{X}-i Y \partial_{Y}+(m+1) \lambda\right) B$ then $B$ is majorized by $(m+1)^{-1} \kappa^{-1} A$.

A fortiori, $\widetilde{g}_{m}$ is majorized by $\kappa^{-m-1} 2^{2 m+2} M^{2 m+2}\left(1+\kappa^{-1}\right)^{m+1}(1-$ $M v)^{-1}\left(1-M\left(1+\kappa^{-1}\right) v\right)^{-2 m-3}$. Suppose that $\varphi_{m^{\prime}, i^{\prime}}$ is majorized by $K_{m^{\prime}, i^{\prime}} v^{i^{\prime}}$ for all $i^{\prime}$ when $m^{\prime}<m$ and for all $i^{\prime}<i$ when $m^{\prime}=m$ and that $K_{m}(v)=\sum K_{m, i} v^{i}$, then (7) implies that $\varphi_{m, i}$ is majorized by $K_{m, i} v^{i}$, where $K_{m, i}$ is the coefficient of $v^{i}$ in

$$
\frac{M \kappa^{-1} v}{1-M v} K_{m}(v)+\frac{M \kappa^{-1}}{m(1-M v)} K_{m-1}^{\prime}(v)+\frac{2^{2 m} M^{2 m}\left(1+\kappa^{-1}\right)^{m} \kappa^{-m}}{(1-M v)\left(1-M\left(1+\kappa^{-1}\right) v\right)^{2 m+1}} \text {. }
$$

Thus we can choose

$$
K_{m}(v)=\frac{M \kappa^{-1}}{m\left(1-M\left(1+\kappa^{-1}\right) v\right)} K_{m-1}^{\prime}(v)+\frac{2^{2 m} M^{2 m}\left(1+\kappa^{-1}\right)^{m} \kappa^{-m}}{\left(1-M\left(1+\kappa^{-1}\right) v\right)^{2 m+2}} .
$$


Hence, if we take $M$ large enough so that $\widetilde{\phi}_{0}$ is majorized by $K_{0}(u)=$ $\left(1-M\left(1+\kappa^{-1}\right) v\right)^{-2}$, then $K_{m}(u)=2^{2 m+1} M^{2 m}\left(1+\kappa^{-1}\right)^{m} \kappa^{-m}(1-$ $\left.M\left(1+\kappa^{-1}\right) v\right)^{-2 m-2}$, hence $\widetilde{\phi}$ is majorized by $\sum K_{m}(u) Z^{m}$ which is clearly convergent.

Theorem 3. Consider three dimensional Lotka-Volterra system (6).

1) The origin has one analytic first integral of the form $\phi=x y(1+$ $O(x, y, z))$ if and only if $a b=e d$.

2) The origin is integrable if and only if

$$
a b-d e=0, \quad d h-b(i \lambda(a-d)+g)=0 .
$$

Proof: Necessary conditions are obtained as explained above. To prove sufficiency we make use of Lemma 2 above.

1) If $a b-e d=0$ then, restricting to $z=0$ we obtain a vector field

$$
X_{0}=x(i+a x+b y) \frac{\partial}{\partial x}+y(-i+d x+e y) \frac{\partial}{\partial y} .
$$

When either $a$ or $e$ are non-zero then this vector field has an invariant algebraic surface $F=1-i a x+i e y$ with cofactor $a x+e y$, and also the surfaces $x=0$ and $y=0$ with cofactors $i+a x+b y+c z$ and $-i+d x+e y+f z$ respectively. Thus the system has a first integral $\phi_{0}=x y F^{k}$, where $k=-1-d / a(a \neq 0)$ or $k=-1-b / e(e \neq 0)$.

If $a=e=0$ there is an exponential factor $E=e^{i(d x-b y)}$ with cofactor $-d x-b y$ and hence a first integral $\phi_{0}=x y E$.

From Lemma 2, we can therefore construct a first integral $\phi$ of the original system (6).

2) We seek a first integral of the form $y^{\lambda} z^{i} e^{-\psi}$ for some analytic $\psi$. Such an integral exists if and only if we can solve the equation

$$
\dot{\psi}=(\lambda d+i g) x+(\lambda e+i h) y+(\lambda f+i k) z .
$$

From Lemma 2, we can solve this problem if and only if there exists a function $\psi_{0}(x, y)$ such that

$$
X_{0}\left(\psi_{0}\right)=(\lambda d+i g) x+(\lambda e+i h) y .
$$

If either $b$ or $d$ are non-zero, then we let $r=a / d(d \neq 0)$ or $r=e / b$ $(b \neq 0)$. We let $\xi=\ln (1+i r(-d x+b y)) / r$ for $r \neq 0$ and $\xi=i(-d x+b y)$ for $r=0$. Then $X_{0}(\xi)=(d x+b y)$ and so we can choose $\psi_{0}=s \xi$, where we take $s=\lambda+i g / d$ if $d \neq 0$ or $s=\lambda e / b+h / b$ if $d=0$ and $b \neq 0$ (whence $g=a=0$ ). 
If $b=d=0$ then we can choose

$$
\psi_{0}=(\lambda d+i g)(1 / a) \log (1-i a x)+(\lambda e+i h)(1 / e) \log (1+i e y),
$$

where we can replace $(1 / a) \log (1-i a x)$ by $-i x$ if $a=0$ and similarly $(1 / e) \log (1+i e y)$ by $i y$ when $e=0$ in the expression above.

\section{Systems with $(i-1:-i-1: 2)$-resonance}

In this section we shall study the local integrability of the origin for the three dimensional Lotka-Volterra equation,

$$
\begin{aligned}
& \dot{x}=P=x(i-1+b y+c z), \\
& \dot{y}=Q=y(-i-1+d x+e y+f z), \\
& \dot{z}=R=z(2+g x+h y) .
\end{aligned}
$$

The assumption that $a=k=0$ from (1) is a somewhat arbitrary choice, but was chosen to bring the computations to a manageable form. It would be interesting to compute the integrability conditions for the general case of arbitrary $a$ and $k$, but this appears to require much more computational power.

As in the previous case, we will give necessary conditions for the origin of (8) to have one analytic first integral of the form $x y z(1+O(x, y, z))$ and also for the existence of a second first integral of the form $x^{2} z^{1-i}(1+$ $O(x, y, z))$. The computations were carried out in Maple up to terms in $(x y z)^{5}$.

The proof of sufficiency is again handled case by case. However, in the case of one first integral, we have three cases which are are unable to give a complete explanation of why these first integrals exist. This is surprising since the conditions themselves seem very simple. In particular, all three have explicit expressions for an inverse Jacobi multiplier.

Theorem 4. Consider three dimensional Lotka-Volterra system (8). The origin is integrable if an only if one of the following conditions hold:

1) $b=h=2 g f+(1+i) d(f+i c)=0$,

2) $f=c=0$,

3) $g=d=0$,

4) $b+(i+1) h=d=e+h i=f=0$,

5) $h=b=e=0$,

6) $e=2 b f-(1+i) c h+(1-i) f h=2 c d+(1+i) c g-(1-i) f g=$ $2 b d+(1+i) b g+(1-i) d h=0$.

Furthermore, a necessary condition that the origin has an analytic first integral of the form $\phi=x y z(1+O(x, y, z))$ is that either 1), 2) or 3) hold above, or one of the following conditions hold: 
4') $f=d=0$,

5') $e=0$,

6') $f+c=g+d=0$.

The sufficiency of conditions 1)-3) follows immediately from their integrability.

Conjecture 5. We conjecture that the conditions 4'), 5') and 6') are also sufficient conditions for one analytic first integral, but are unable to prove this at the moment.

Proof: The necessity of the conditions is proved as indicated above. We shall treat the proofs of sufficiency case by case.

Case 1: The equations for $\dot{x}$ and $\dot{z}$ are decoupled from the $\dot{y}$ equation and have eigenvalues in the Poincaré domain with no resonances possible. Hence we can find linearizing transformations $(X, Z)=(x(1+$ $O(x, z)), z(1+O(x, z)))$ which bring the system to the form

$\dot{X}=(i-1) X, \quad \dot{y}=y(-1-i+d x(X, Z)+e y+f z(X, Z)), \quad \dot{Z}=2 Z$.

We now seek an invariant analytic surface of the form $\alpha(X, Z)+$ $y \beta(X, Z)=0$ such that

$\frac{d}{d t}(\alpha(X, Z)+\beta(X, Z) y)=(\alpha(X, Z)+\beta(X, Z) y)(d x(X, Z)+e Y+f z(X, Z))$.

The third equation can then be linearized with the substitution $Y=$ $y /(\alpha(X, Z)+\beta(X, Z) y)$.

These conditions reduce to

$$
\dot{\alpha}=\alpha(d x(X, Z)+e y+f z(X, Z)), \quad \dot{\beta}-(i+1) \beta=\alpha e .
$$

Since

$$
\frac{d}{d t} \sum_{r, s \geq 0} a_{r, s} X^{r} Z^{s}=\sum_{r, s \geq 0}(r(i-1)+2 s) a_{r, s} X^{r} Z^{s} .
$$

The first equation can be solved uniquely term by term and is clearly convergent. The second equation can be solved likewise if and only if the coefficient of $X Z$ in $\alpha$ is zero. However, a small computation shows that this condition is just $2 g f+(1+i) d(f+i c)=0$.

Case 2: In this case the critical point at the origin for the first and second equation are in the Poincaré domain and hence is linearizable using a change of coordinates $(X, Y)=(x(1+O(x, y)), y(1+O(x, y)))$ that gives

$$
\dot{X}=(i+1) X, \quad \dot{Y}=-(i+1) Y, \quad \dot{z}=z(2+g x(X, Y)+h y) .
$$


Suppose there exists a function $\gamma$ such that $\dot{\gamma}=g x(X, Y)+h y(X, Y)$. The transformation $Z=z e^{-\gamma}$ will linearize third equation. Writing $g x(X, Y)+h y(X, Y)=\sum_{n+m>0} a_{n, m} X^{n} Y^{m}$, we see that

$$
\gamma=\sum_{n+m>0} \frac{a_{n, m}}{(i-1) n-(i+1) m} X^{n} Y^{m},
$$

which is clearly convergent.

Case 3: This case is effectively the same as the previous case, except that now the second and third equations are in the Poincaré domain and therefore there exists a change of variables $(Y, Z)=(y(1+O(y, z)), z(1+$ $O(y, z)))$ such that

$$
\dot{x}=x(i-1+b y(Y, Z)+c z(Y, Z)), \quad \dot{Y}=-(i+1) Y, \quad \dot{Z}=2 Z .
$$

We seek a function $\gamma$ such that $\dot{\gamma}=b y(Y, Z)+c z(Y, Z)$. Then the transformation $X=x e^{-\gamma}$ gives $\dot{X}=(i-1) X$. To find such a function, we write $b y(Y, Z)+c z(Y, Z)=\sum_{n+m>0} a_{n, m} Y^{n} Z^{m}$, then we have

$$
\gamma=\sum_{n+m>0} \frac{a_{n, m}}{2 m-(i+1) n} Y^{n} Z^{m},
$$

which gives a convergent expression for $\gamma$.

Case 4: When $e \neq 0$

$\dot{x}=x(i-1-(i+1) h y+c z), \quad \dot{y}=y(-i-1-i h y), \quad \dot{z}=z(2+g x+h y)$.

In this case the system has an invariant algebraic plane $\ell=1+\frac{i+1}{2} h y=0$ and an exponential factor $E=\exp \left(\frac{g x-c y}{1+\frac{(i+1)}{2} h y}\right)$ with cofactors $L_{\ell}=-i h y$ and $L_{E}=(i-1) g x-2 c z$ producing a first integral $\phi=x^{2} z^{1-i} \ell^{i-3} E$ and inverse Jacobi multiplier $M=x y z \ell$. Theorem 1 then guarantees the existence of a second first integral of the form $\phi^{\prime}=1+O(x, y, z)$, which must be analytic. Consideration of the first non-constant terms in this expansion imply that $\phi^{\prime}-1=(x y z)^{k}(c+O(x, y, z))$ for some $c \neq 0$ and therefore the system is integrable.

Case 5: The equations for $\dot{x}$ and $\dot{z}$ are decoupled from the $\dot{y}$ equation so we can find linearizing transformations $(X, Z)=(x(1+O(x, z)), z(1+$ $O(x, z)))$ which bring the system to the form

$$
\dot{X}=(i-1) X, \quad \dot{y}=y(-1-i+d x(X, Z)+f z(X, Z)), \quad \dot{Z}=2 Z .
$$

We seek a function $\gamma$ such that $\dot{\gamma}=d x(X, Z)+f z(X, Z)$. Then the transformation $Y=y e^{-\gamma}$ gives $\dot{Y}=-(i+1) Y$. 
If $d x(X, Z)+f z(X, Z)=\sum_{n+m>0} a_{n, m} X^{n} Z^{m}$, then we have

$$
\gamma=\sum_{n+m>0} \frac{a_{n, m}}{(i-1) n+2 m} X^{n} Z^{m}
$$

which gives a convergent expression for $\gamma$.

Case 6: The equations guarantee that the cofactors of $x, y$ and $z$ are linearly dependent, so there exists a first integral of the form $x^{\alpha} y^{\beta} z^{\gamma}$. We also have an inverse Jacobi multiplier $x y z$. Theorem 1 therefore guarantees the existence of a first integral of the form $\phi^{\prime}=1+O(x, y, z)$, which must be analytic. Consideration of the first non-constant terms in this expansion imply that $\phi^{\prime}-1=(x y z)^{k}(c+O(x, y, z))$ for some $c \neq 0$ and therefore the system is integrable.

\section{Acknowledgements}

We would like to thank the referees for their careful reading and comments on the original manuscript which saved us from a number of errors and improved the presentation.

\section{References}

[1] W. AzIz, Integrability and linearizability of three dimensional vector fields, Submitted.

[2] W. Aziz and C. Christopher, Local integrability and linearizability of three-dimensional Lotka-Volterra systems, Appl. Math. Comput. 219(8) (2012), 4067-4081. DOI: 10.1016/j.amc.2012.10. 051.

[3] W. Aziz, C. Christopher, J. Llibre, and C. Pantazi, Local analytic first integrals of three-dimensional Lotka-Volterra systems with $(3:-1: 2)$-resonance, Submitted.

[4] V. V. Basov and V. G. Romanovski, First integrals of a threedimensional system in the case of one zero eigenvalue, J. Phys. A 43(31) (2010), 8 pp. DOI: 10.1088/1751-8113/43/31/315205.

[5] L. R. Berrone and H. Giacomini, Inverse Jacobi multipliers, Rend. Circ. Mat. Palermo (2) 52(1) (2003), 77-130. DOI: 10.1007/BF02871926.

[6] M. Bobieński And H. ŻoŁA̧DeK, The three-dimensional generalized Lotka-Volterra systems, Ergodic Theory Dynam. Systems 25(3) (2005), 759-791. DOI: 10.1017/S0143385704000902.

[7] L. CAIRó, Darboux first integral conditions and integrability of the 3D Lotka-Volterra system, J. Nonlinear Math. Phys. 7(4) (2000), 511-531. DOI : 10.2991/jnmp.2000.7.4.7. 
[8] L. Cairó and J. Llibre, Darboux integrability for 3D LotkaVolterra systems, J. Phys. A 33(12) (2000), 2395-2406. DOI: 10.1088/0305-4470/33/12/307.

[9] Y. T. Christodoulides and P. A. Damianou, Darboux polynomials for Lotka-Volterra systems in three dimensions, J. Nonlinear Math. Phys. 16(3) (2009), 339-354. DOI: 10.1142/ S1402925109000261.

[10] C. Christopher and C. Rousseau, Normalizable, integrable and linearizable saddle points in the Lotka-Volterra system, Qual. Theory Dyn. Syst. 5(1) (2004), 11-61. DOI: 10.1007/BF02968129.

[11] F. Gonzalez-Gascon and D. Peralta Salas, On the first integrals of Lotka-Volterra systems, Phys. Lett. A 266(4-6) (2000), 336-340. DOI : 10.1016/S0375-9601(00)00011-6.

[12] S. Gravel and P. Thibault, Integrability and linearizability of the Lotka-Volterra system with a saddle point with rational hyperbolicity ratio, J. Differential Equations 184(1) (2002), 20-47. DOI: 10.1006/jdeq. 2001.4128 .

[13] C. Liu, G. Chen, And C. Li, Integrability and linearizability of the Lotka-Volterra systems, J. Differential Equations 198(2) (2004), 301-320. DOI : 10.1016/S0022-0396 (03) 00196-7.

[14] J. Moulin Ollagnier, Liouvillian integration of the LotkaVolterra system, Qual. Theory Dyn. Syst. 2(2) (2001), 307-358. DOI : $10.1007 / \mathrm{BF} 02969345$.

Waleed Aziz:

Department of Mathematics

College of Science

University of Salahaddin

Kurdistan Region

Iraq

E-mail address: waleed.aziz@plymouth.ac.uk

Colin Christopher:

School of Computing and Mathematics

Plymouth University

Plymouth, Devon, PL4 8AA

UK

E-mail address: C.Christopher@plymouth.ac.uk 